\title{
Nachwuchswissenschaft, la jeune recherche et les « Mots de l'histoire"
}

\section{Anna Karla}

\section{OpenEdition}

\section{Journals}

Édition électronique

URL : http://journals.openedition.org/ifha/463

DOI : $10.4000 /$ ifha.463

ISSN : 2198-8943

\section{Éditeur}

IFRA - Institut franco-allemand (sciences historiques et sociales)

\section{Édition imprimée}

Date de publication : 30 septembre 2012

Pagination : 204-213

ISSN : 2190-0078

\section{Référence électronique}

Anna Karla, "Nachwuchswissenschaft, la jeune recherche et les " Mots de l'histoire » », Revue de l'IFHA [En ligne], 4 | 2012, mis en ligne le 14 février 2013, consulté le 01 mai 2019. URL : http:// journals.openedition.org/ifha/463 ; DOI : 10.4000/ifha.463

Ce document a été généré automatiquement le 1 mai 2019.

(CIFHA 


\title{
Nachwuchswissenschaft, la jeune recherche et les « Mots de l'histoire »
}

\author{
Anna Karla
}

Parler en tant que représentante des jeunes chercheurs lors du bilan des «Mots de l'histoire » veut dire deux choses. En premier lieu, c'est une invitation à dépasser l'horizon personnel d'une participation au séminaire de quelques années seulement pour évoquer, de manière plus globale, les souvenirs et impressions des jeunes participants du séminaire. Deuxièmement, c'est une incitation à parler au nom d'un groupe, celui des jeunes chercheurs ou des Nachwuchswissenschaftler, dans un cadre propice à l'analyse des termes communément utilisés en histoire. Pourtant, en se penchant sur ces « Mots », il est rapidement apparu qu'il s'agissait en réalité de catégories diffuses et problématiques. Pour mieux me tirer d'affaire, j'ai donc choisi la voie de l'empirisme. Dans une petite enquête ${ }^{1}$ quoique peu représentative, des participants ont ainsi parlé de leurs souvenirs individuels du séminaire. Dans un cercle plus large de jeunes chercheurs français et allemands, ceux qui ont participé à l'enquête ont également donné leurs avis sur ce qu'ils entendaient par « jeune chercheur » ou par Nachwuchswissenschaftler.

\section{1) «Les Mots de l'histoire » vus par les jeunes chercheurs - un bilan}

La plupart des jeunes participants au séminaire provenaient des programmes partenaires de l'EHESS, des universités parisiennes ou de l'Université franco-allemande, à savoir souvent les programmes de master binationaux et les collèges doctoraux francoallemands. Grâce à une coopération logistique avec l'Institut historique allemand depuis 2008, un bon nombre de boursiers de l'IHA purent aussi découvrir le séminaire et y participèrent pendant la durée de leur séjour à Paris.

Le temps fort de la participation active des jeunes chercheurs au séminaire fut sans doute la Journée Junior organisée principalement par Franziska Heimburger (EHESS). Les participants provenaient des institutions partenaires. Sur le modèle du séminaire, des 
étudiants en master et des doctorants allemands et français étudièrent ainsi des couples notionnels sur les thématiques suivantes : "Repräsentationen/représentation ", "Land und Herr/Le maitre et son territoire/son pays ", "Selbstzeugnisse/Écrits du for privé » et " Revolution/Révolution ». Dans un deuxième temps de la journée fut évoquée l'organisation de la recherche en sciences sociales et humaines dans le domaine franco-allemand.

Les participants à la Journée Junior et au séminaire soulignèrent la «plus-value » de ce format, notamment pour les jeunes chercheurs. Ainsi était-il possible, dans ce cadre, de surpasser - ou du moins de thématiser - les frontières nationales :

«Ce que j'ai aimé en particulier, c'était la combinaison des concepts allemands et français et, par la suite, le fait de rassembler des chercheurs marqués par différents courants historiographiques nationaux."

"Il était très intéressant, de percevoir, dans une confrontation directe, des styles de recherche et de rhétorique différents. »

La participation au séminaire apparut ainsi comme un enrichissement, grâce auquel les jeunes chercheurs trouvaient des suggestions concrètes pour leurs propres recherches :

«Pour moi, en tant qu'Allemand en France, c'était l'un des séminaires les plus instructifs, car il avait pour objet un de mes défis quotidiens : l'(in)traductibilité des concepts, avec lesquels je travaille. »

Pour les jeunes chercheurs, un autre point fort du séminaire tenait à sa dimension interdisciplinaire. Car, même si le travail interdisciplinaire n'est pas propre à l'EHESS, les participants appréciaient l'ouverture concrète à d'autres disciplines dans le séminaire, et qu'ils regrettaient de ne pas voir autant à l'oeuvre dans la recherche allemande.

\section{2) Les « Mots de l'histoire » et l'organisation de la recherche scientifique pour les jeunes chercheurs franco-allemands}

Ce bilan positif est en grande partie lié à la conjoncture scien-tifique assez remarquable dans lequel s'inscrivait le séminaire. De plus en plus institutionnalisé au cours des huit années de son existence, le séminaire revêtit une importance structurelle. Il rassemblait en effet, de manière régulière et durable, des étudiants des programmes de master binationaux, des doctorants des collèges franco-allemands, ainsi que des boursiers et des chercheurs de l'Institut historique allemand de Paris. Sans pouvoir indiquer de chiffres exacts, il semble que les Allemands furent un peu plus nombreux que les Français. Pour les participants de la Journée Junior, un équilibre national « fut peut-être un peu difficile à réaliser ». Il n'en demeure pas moins que les vendredi matins à l'Hôtel-Duret-de-Chevry étaient de belles occasions de se réunir, et servaient de point de rencontre pour tous ceux qui travaillaient, dans leurs thèses ou dans leur mémoire de master, entre, sur ou avec la France et l'Allemagne.

Mais les « Mots de l'histoire » étaient aussi un lieu d'échanges et de rencontres sur un plan scientifique. Le séminaire était en ce sens le lieu par excellence où se jouait de vivo ce que les cursus universitaires bi- et internationaux visent à encourager. Le fait en premier lieu qu'on n'enquête pas « sur » l'autre, mais qu'on travaille avec lui; qu'il est nécessaire de remettre en question ses habitudes et ses prétendues certitudes historiographiques; qu'enfin c'est de la confrontation et de la comparaison avec le non-semblable que peut surgir le transfert des concepts et des problématiques. En somme, pour reprendre les 
termes d'un participant, il s'agissait là d'une "mise en pratique réussie d'un véritable échange scientifique franco-allemand».

Le séminaire apporta une contribution fondamentale au dialogue franco-allemand dans le domaine de la recherche. "Penser les différences", titre du collège doctoral francoallemand de l'université Humboldt de Berlin et de l'EHESS, part d'une approche semblable. Les membres du collège ont réfléchi, lors de leur dernière rencontre fin mai 2012 à Berlin, sur des couples notionnels tels que « Globalgeschichte/Histoire globale ", " Geschichtspolitik/Usages politiques du passé », "Übersetzen/Traduire ». Le schéma des "Mots de l'histoire " se perpétue ainsi au-delà du cadre même du séminaire. De fait, celui-ci contribua à établir une coopération scientifique franco-allemande qui ne se résumait pas à des étiquettes politiquement correctes ou à des frais de mobilité. Durant les séances des « Mots de l'histoire » on pouvait voir, en quelque sorte, cette coopération à l'œuvre, avec de véritables échanges scientifiques visant la compréhension réciproque, la connaissance de l'autre, le croisement des traditions historiographiques et méthodologiques. L'une des participantes soulignait à ce propos :

« Par rapport à mes propres recherches, je n'ai pas vraiment eu l'impression d'avoir accès à des nouvelles thématiques, mais plus à un bilan et à une clarification rassurante dans le flou conceptuel et institutionnel qui règne parfois dans mes propres recherches. "

Ce qui pouvait sembler évident pour les participants de longue date, se présentait donc comme un processus d'apprentissage nécessaire à chaque nouvelle génération d'étudiants en master et de doctorants franco-allemands. Les « Mots de l'histoire » eurent, dans cet apprentissage, une place incontestée et primordiale.

\section{3) Retour sur les mots ou qu'est-ce que le Nachwuchswissenschaftler?}

Dans le cadre universitaire et scientifique actuel, le terme de jeune chercheur ou de Nachwuchswissenschaftler est omniprésent. Rien que par son emploi récurrent, il mérite une attention particulière dans le cadre des « Mots de l'histoire ». Car si le séminaire a traité principalement de concepts historiographiques et des mots des sources, cette dernière séance peut constituer l'occasion d'attirer l'attention sur ces termes qu'on emploie pour nous désigner - des termes qui peuvent sembler anodins au premier regard, mais qui influent de fait sur la manière de dire et de faire des travaux scientifiques. Il s'agit donc de proposer un repérage, en partant d'une question toute simple : qu'est ce qu'un Nachwuchswissenschaftter? Les réflexions suivantes se réfèrent en premier lieu au concept allemand, mais elles montreront qu'une comparaison en couple notionnel entre le Nachwuchswissenschaftler et le jeune chercheur s'impose.

Le Nachwuchs (littéralement traduit : l'enfant, la relève) constitue actuellement l'un des concepts clés dans l'espace universitaire allemand. Pour désigner ce même phénomène, on parle en France de jeunes chercheurs et de la jeune recherche, les pays anglo-saxons utilisant les termes de young academics ou encore d'early career. Comme tout mot de prédilection de l'allemand académique, le Nachwuchs se prête très bien à l'association avec un autre substantif. Le nombre de mots composés qui en résulte est légion: Nachwuchswissenschaft (jeune recherche), Nachwuchswissenschaftlerin (jeune chercheuse), Nachwuchsförderprogramm (programme d'encouragement de la jeune recherche), 
Nachwuchsforschergruppe (groupe de recherche des jeunes chercheurs), Nachwuchstagung (conférence des jeunes chercheurs)... On pourrait facilement allonger la liste.

Mais qui désigne-t-on par cette catégorie? Il semble difficile d'établir une définition du Nachwuchswissenschaftler. Deux institutions parmi les plus influentes en ce qui concerne la relève scientifique sont manifestement en désaccord sur le moment biographique à partir duquel quelqu'un entre dans le parcours de la Nachwuchswissenschaft pour devenir ainsi un jeune chercheur. Sur son site internet, le Deutscher Hochschulverband (DHV, association des enseignants du supérieur allemands) déclare de manière quasi définitoire: «Êtesvous un Nachwuchswissenschaftler (en cours de préparation d'une habilitation à diriger des recherches, professeur junior, chef d'un groupe de recherche et de travail scientifique, Privatdozent ${ }^{2}$ ou dans une position comparable)?». Pour ensuite ajouter sur un ton chaleureux : « Dans ces cas-là, vous êtes les bienvenus au Hochschulverband. $»^{3}$ On apprend ici que le statut de jeune chercheur commence au début de l'habilitation ou en tant que professeur junior. Il en est tout autrement de la Deutsche Forschungsgemeinschaft (DFG, Fondation allemande de la recherche). Son magazine en ligne «Karrierewege " (parcours de carrière) explique: "L'encouragement du Nachwuchs scientifique est au cœur des préoccupations de la DFG. C'est pour cela qu'elle invite des élèves intéressés à nouer de bonne heure un contact avec la science. » Outre les informations sur les programmes de financement scientifique pour les post-doctorants et les jeunes professeurs (programme Emmy-Noether, professorat Heisenberg), la rubrique sur le site internet du " Wissenschaftlicher Nachwuchs» (relève scientifique) comporte une offre pédagogique diverse, telle que «Wissenschaft für Kids» (Science pour les enfants), «Experimentieren in Forschungszentren " (expériences dans les centres de recherches ») et «Dem Wissenschaftler über die Schulter schauen » (regarder au dessus de l'épaule du scientifique). ${ }^{4}$

À l'évidence, il existe différentes datations dans le domaine du Nachwuchs. On pourrait argumenter que le Hochschulverband part d'un besoin de recrutement concret tandis que la DFG tiendrait au contraire à l'idéal démocratique d'une Bildung pour tous dès le départ. On peut néanmoins constater deux choses. Premièrement, le terminus ante quem semble fixe. N'est plus Nachwuchswissenschaftler qui devient titulaire d'une chaire. Dans ce contexte on a utilisé le terme plus neutre de "nicht-professoralen Wissenschaftler $"^{5}$ (scientifique non-professeur). En Allemagne, le métier de scientifique commence traditionnellement avec la nomination en tant que professeur, même si le groupe des scientifiques non-professeurs forme la grande majorité du personnel universitaire. Deuxièmement, le terminus post quem - c'est à dire le moment à partir duquel on peut être un Nachwuchswissenschaftler - est loin d'avoir une définition univoque. D'habitude, on parle souvent du diplôme universitaire et l'on considère ainsi les doctorants comme des jeunes chercheurs. Pourtant, avec le financement accru des postes et des bourses pour post-doctorants, la thèse semble devenir une simple carte d'entrée pour le statut de jeune chercheur. En France, le couple notionnel de doctorants et jeunes chercheurs s'emploie souvent non comme un équivalent, mais dans une succession temporelle. Le vrai Nachwuchswissenschaftler est ainsi - comme le suggérait le « Hochschulverband » allemand le chercheur qui a terminé sa thèse, mais qui n'a pas encore été nommé pour un poste de durée indéterminée (c'est-à-dire, dans le contexte allemand, un poste de professeur). Au regard de la rhétorique de la $\mathrm{DFG}$, la relève scientifique amorce ses explorations à un tout autre moment de la vie, à savoir dès l'âge de l'école primaire, voire même à l'école maternelle ou à la crèche. Le mot-clé du "Lebenslanges Lernen " (apprentissage tout au long de la vie) est projeté ici non sur la vieillesse mais sur la jeunesse. L'esprit scientifique 
se présente comme quelque chose qu'on ne peut jamais acquérir trop tôt et qui mérite la protection et l'encouragement politique et financier.

Le stade initial de la jeune recherche se présente donc comme diffus. Pour le terme allemand, on peut en trouver la raison dans le noyau étymologique du concept. Le Nachwuchs se présente comme métaphore biologique. Elle rappelle les processus physiologiques de croissance, et se fonde sur l'idée du Nachwachsen (repousser), du Hineinwachsen (de grandir dans quelque chose, c'est-à-dire de s'adapter), d'atteindre un statut du Er-Wachsensein (d'adulte). Au sein des sociétés, ce vocabulaire renvoie habituellement au champ sémantique familial. La progéniture a besoin de soin, d'éducation et d'assistance. Sur une échelle sociale plus large, le terme Nachwuchs vise le champ sémantique de la génération ou de la cohorte. Ce groupe de même âge se trouve au moment $\mathrm{x}$ dans un stade de croissance, et nécessite de ce fait une attention particulière et adaptée. On peut d'ailleurs se demander si l'emploie fréquent du terme Nachwuchswissenschaftler dans la recherche des années 1970 et 1980 en RFA ne s'expliquerait pas par cette dimension sociale large. Les colloques pour la jeune recherche y figuraient en effet parmi les instruments typiques de l'organisation inter-scientifique. ${ }^{6}$ $\mathrm{Au}$ final, ce qui unit tous ces domaines, c'est l'idée qu'il s'agit de quelque chose en devenir, d'un état en suspens, d'un statut en attente de son stade de maturité et de vie d'adulte.

Le Nachwuchswissenschaftler s'avère donc comme un concept fondamentalement téléologique. C'est pour cela qu'il correspond parfaitement à une rhétorique de politique éducative qui tend à sublimer les limites de croissance de la société postindustrielle par le renforcement du "Wissenschaftsstandort Deutschland» (Allemagne - haut-lieu de la recherche et du savoir). Quand on mobilise de l'argent pour l'encouragement du Nachwuchs, on part de l'idée optimiste d'un progrès positif. On pourrait même considérer que parler de relève correspond aux objectifs internes de la science moderne. En fin de compte, il s'agit là d'accumuler des connaissances et de l'expérience, de savoir toujours plus, de réinterroger constamment d'anciennes habitudes, de faire grandir de nouvelles idées. Selon ses activités et sa disposition mentale, chaque scientifique reste donc en quelque sorte durant toute sa carrière dans un processus de croissance et de perfectibilité.

Là où s'impose, sous le voile de la téléologie, sa composante biologique, le terme commence à poser problème. On constate un clivage entre la théorie et la pratique : l'idée de croissance et de progrès ne correspond pas toujours aux données réelles du marché du travail universitaire. L'optimisme du progrès se trouve donc trop facilement transformé en social-darwinisme affiché. Qui grandira plus vite aura plus de chance d'arriver au but. Qui poussera trop lentement ou dans une fausse direction sera obligé de se « déplanter ». Au lieu de vivre une solidarité intergénérationnelle ou une attention familiale, il s'agit plutôt de contourner ou de sauter des étapes du Nachwuchs, de commercialiser ses atouts personnels et de propager sa propre performance scientifique, bref de prendre un chemin de carrière individuel voire individualiste. À une époque où la recherche est financée sur des critères d' "excellence », on ne s'étonne plus que l'ouverture et la démocratisation des universités aillent de pair avec des stratégies individualistes de plus en plus flagrantes.

La rhétorique optimiste du Nachwuchs se trouve donc en contradiction avec les conditions concrètes de croissance. Il faut être un bon idéaliste pour croire en la perspective de se retrouver, un jour, parmi les scientifiques adultes ou majeurs. Il y a là une tension latente 
entre un excès rhétorique d'un côté et le chemin réel de la carrière au sein du système universitaire de l'autre. C'est en cela qu'il est certainement possible de constater une spécificité du système allemand. Car si on peut se débarrasser de son statut de jeune en Angleterre à partir d'un poste de lecturer, en France avec le statut de maître de conférences, l'adolescence scientifique dure sensiblement plus longtemps en Allemagne.

La recherche en sociologie académique s'intéresse de plus en plus à se sujet - ce qui indique déjà sa potentielle problématique. Au cours des dernières années, le Nachwuchswissenschaftler est devenu une catégorie réflexive (sans pour autant qu'on ait porté une attention particulière au terme même). En témoignent plusieurs études en sociologie consacrées au "Hürdenlauf» (course de haies) ou aux "Karrierestufen junger Wissenschaftlerinnen und Wissenschaftler $»^{7}$ (étape de carrière des jeunes chercheuses et jeunes chercheurs). Le débat pourtant n'est pas nouveau. Le début d'une véritable «Krise des wissenschaftlichen Nachwuchses » (crise des jeunes chercheurs) date des années 1970. À cette époque, l'ouverture des universités à un nombre considérable d'étudiants rendait impossible l'ancien rapport entre un professeur et son élève ${ }^{8}$. Dès 1986, une étude commandée par le Hochschulverband tirait la conclusion désenchantée suivante: «Il n'existe pas de recette miracle pour la solution du problème complexe et de longue durée du Nachwuchs. $»^{9}$

En fin de compte, il faut prêter la voix à ceux qui se sentent personnellement concernés par cette désignation. Biographiquement, ces personnes se trouvent en général quelque part entre la thèse et les entretiens pour les rares postes de professeurs. Ils se situent entre la fin de la vingtaine et le début de la quarantaine. Leur rapport au terme de Nachwuchswissenschaftler est pour la plupart des cas cynique. Se considèrent-ils comme des Nachwuchswissenschaftler? Ni oui, ni non. Oui, parce que dans le cadre scientifique, on fait figure d'un Nachwuchswissenschaftler. Non, car il s'agit là d'une désignation venue de l'extérieur. On ne pourrait pas parler de Nachwuchs sans un soupçon d'ironie. Le terme suggère tout d'abord " élevage » et « soin ». Mais, contrairement à l'ancien rapport entre professeur et élève, on le perçoit comme une formule toute faite sans responsabilité aucune vis-à-vis des jeunes chercheurs. Alimenter le Nachwuchs est donc considéré comme une manière dépersonnalisée de diffuser de l'argent selon un principe de saupoudrage. En comparaison du jeune chercheur français et du young academic angloaméricain, le Nachwuchswissenschaftler allemand présenterait même une dégradation de son propre travail de recherche. Entre trente et quarante ans, des attributs comme «jeune » ou "young " peuvent à la limite flatter. En revanche, le mot composite en allemand suggérerait qu'on n'est pas encore un scientifique mais qu'on est en passe de le devenir. Pour ceux qui ont dépassé les 35 ans, l'étiquette du Nachwuchs devient une simple "discrimination». Ce qui unit ces prises de paroles touche à la volonté de distinguer entre un positionnement lié à l'âge et le travail scientifique proprement dit. Car même si on est étiqueté, dans le système universitaire, en tant que Nachwuchs, on refuse l'idée que ses propres travaux de recherches soient reçus sous ce prédicat.

Ce bref survol soulève un bon nombre de questions. On pourrait s'amuser à comparer les ressemblances et les différences entre le Nachwuchswissenschaftler et le jeune chercheur. On devrait certainement établir des différences entre les chercheurs en sciences humaines et sociales et les chercheurs en sciences naturelles, moins fixés sur le marché de travail universitaire, et du coup moins concernés par l'aspect darwiniste du terme. On devrait finalement poser la question cruciale de toute cette thématique, à savoir le rapport entre ce phénomène biologico-politico-démographique qu'est la Nachwuchswissenschaft et les 
contenus scientifiques produits par ses membres. Quel impact exerce sur la science le fait que des générations entières de chercheurs se trouvent sur des voies professionnelles qui rappellent plus une roue pour hamster qu'une échelle adaptée à la croissance ? Comment s'accorde l'idée d'un apprentissage tout au long de la vie, individuel et diachrone, constitutif de la biographie scientifique, avec des modèles de formation en cohorte, synchrone, et susceptible d'encourager un conformisme thématique? Le Nachwuchswissenschaftler sera-t-il jugé sur sa capacité d'adaptation évolutive ou sur sa trajectoire individuelle? - Autant de questions qui auraient mérité de futures séances des « Mots de l'histoire».

\section{NOTES}

1. Mes remerciements vont particulièrement à Luca Hardt, Marie-Christin Lux, Antoine Odier, Pauline Pujo et Johanna de Schmidt pour leurs impressions du séminaire. Je remercie de manière globale tous ceux qui ont pris part à l'enquête anonyme sur le Nachwuchswissenschaftler et ont enrichi cet exposé par leurs idées et leurs expériences.

2. Le Privatdozent (PD) allemand désigne des chercheurs ayant obtenu une habilitation à diriger des recherches, mais qui ne sont pas titulaires d'une chaire.

3. www.hochschullehrerverband.de/cms1/wissnachwuchs0.html (12.06.2012). Nous traduisons de l'allemand, comme c'est le cas dans la suite de cet article.

4. www.dfg.de/dfg_magazin/wissenschaftliche_karriere/index.html (12.06.2012).

5. Cf. Jürgen Enders, Die wissenschaftlichen Mitarbeiter. Ausbildung, Beschäftigung und Karriere der Nachwuchswissenschaftler und Mittelbauangehörigen an den Universitäten, Frankfurt a. M./New York : Campus, 1996.

6. Cf. les nombreuses entrées sur la Nachwuchswissenschaft issues du système universitaire de la RFA entre 1972 et 1987 dans les catalogues de bibliothèque allemandes.

7. Ina Findeisen, Hürdenlauf zur Exzellenz. Karrierestufen junger Wissenschaftlerinnen und Wissenschaftler, Wiesbaden, 2010.

8. Cf. Enders, Die wissenschaftlichen Mitarbeiter, 9.

9. Ulrich Karpen, Zur Lage des habilitierten wissenschaftlichen Nachwuchses, in: Forum des Hochschullehrerverbands, édité par le Präsidium des Deutschen Hochschullehrerverbandes, vol. 40, décembre 1986, 2. 


\section{AUTEUR}

\section{ANNA KARLA}

Anna Karla est doctorante en cotutelle à l'université Humboldt de Berlin et à l'École des hautes études en sciences sociales. Elle est actuellement boursière de l'Institut historique allemand de Paris. 